\title{
Benign Sacrococcygeal Teratoma in Adults: A Systematic Review of the Literature
}

\author{
Anudeep Yekula ${ }^{1 *}$, Sulekhya Inturu ${ }^{2}$, Samiksha Jain ${ }^{2}$, Anuroop \\ Yekula ${ }^{2}$, Abhinaya Kotha ${ }^{2}$, Anurag Pasula ${ }^{3}$ and Kiran Kumar Yekula ${ }^{4}$ \\ ${ }^{1}$ Department of Neurosurgery, Massachusetts General Hospital and Harvard \\ Medical School, Boston, MA, USA \\ ${ }^{2}$ Government General Hospital, Guntur Medical College, Guntur, India \\ ${ }^{3}$ Government General Hospital, Rangaraya Medical College, Kakinada, India \\ ${ }^{4}$ Kocher's Institute of Medical Sciences, Guntur, India \\ *Corresponding Author: Anudeep Yekula, Department of Neurosurgery, \\ Massachusetts General Hospital and Harvard Medical School, Boston, MA, USA.
}

Received: April 16, 2021

Published: May 25, 2021

(C) All rights are reserved by Anudeep

Yekula., et al.

\begin{abstract}
Introduction: Sacrococcygeal teratomas are tumors derived from embryonic germ cell layers predominantly occurring in pediatric cohorts. They are rare in adults and limited data exists on their clinicopathologic characteristics and their management.

Methods: The aim of the study is to explore the demographic, clinical, radiographic, histopathologic, and the management of SCT in adults through a comprehensive systematic review of literature.

Results: A total of 73 patients with benign sacrococcygeal teratoma were identified (51, 69.8\% female), with a median age of 30.25 years. Patients mostly presented with pelvic pain $(n=37)$ and/or palpable mass $(n=33)$. The majority of the tumors belonged to Altman type IV $(28,56 \%)$. Surgical resection with coccygectomy was almost always curative.

Conclusion: Benign SCTs require multidisciplinary management. Careful evaluation is required in all cases of sacral masses, and prompt differentiation of benign entities from the malignant counterparts is central to the management of these lesions.

Keywords: Sacrococcygeal Teratoma; SCT; Adult; Benign
\end{abstract}

\section{Introduction}

Sacrococcygeal teratomas (SCT) are rare tumor entities derived from pluripotent embryonic germ cell layers at the site of the coccyx. They are uniquely composed of tissue derivatives from one or more primordial germ layers (endoderm, mesoderm, and ectoderm). They commonly occur in neonates with an estimated incidence of 1 in 40,000 births and females with a female to male ratio of $4: 1$ [1]. The majority of the SCTs are identified in prenatal diagnosis, 50\%-70\% within a few days after birth, and about $10 \%$ after age 2 years [2]. However, a very small cohort of patients has been reported to have SCT as an adult in the literature. The true incidence of benign SCT in adults is not known. The clinical presentation, diagnosis, and management strategies are unique in adults and have been poorly studied.
Here, we perform a comprehensive systematic review of literature of adult cases of SCT and discuss the demographic, clinical, radiographic, histopathologic, and management of the disease.

Systematic Literature Review

Methods

We performed a systematic search for all cases of benign SCT in adult patients reported in the literature using the Google Scholar, PubMed, MEDLINE databases since 1960. The results of the search strategies are summarized in figure 1. Inclusion criteria were 1) histologically confirmed cases of benign SCT, 2) occurring in adults (> 16 years age), and 3) English language. Search strings included all possible combinations of the terms "Sacrococcygeal teratoma" 


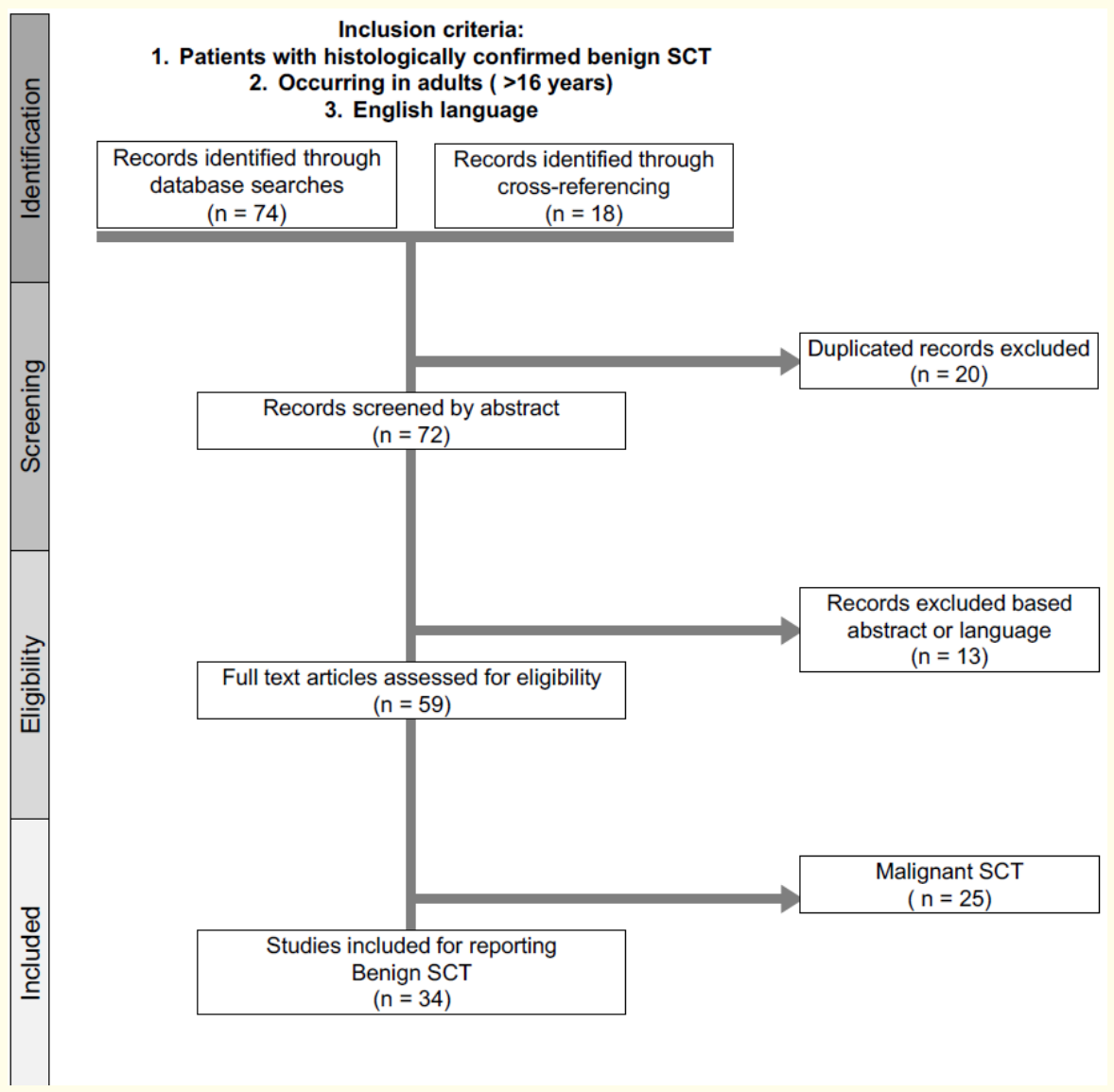

Figure 1: Flow diagram showing the systematic screening of studies for the analysis of adult cases of benign SCT.

with the terms "adult", "benign", and "malignant". We screened abstracts and full-text articles to identify reports that passed our inclusion criteria. We also screened the references listed in each study to identify additional publications. Malignant SCT cases were included in our follow-up review. We excluded cases that lacked case-level descriptions of clinical presentation, diagnosis, and management. We followed Preferred Reporting Items for Systematic Reviews and Meta-Analysis (PRISMA) criteria for the systematic review of the literature.

\section{Results}

We performed a systematic literature review of benign (Table 1) cases of SCT in adults. A total of 73 patients were reported across 34 studies including case reports, case series, and retrospective studies [2-28]. The ages ranged from 17 to 74 years, with a median age of 30.52 years. Gender was female in $69.8 \%(n=51)$ of patients. The median duration of symptoms was 6 years, with some patients having symptoms since birth. The majority presented with a combination of pelvic pain $(n=39)$ and/or mass $(n=33)$. Changes in bowel $(\mathrm{n}=14)$ and bladder $(\mathrm{n}=12)$ function were often noted. Ulceration, drainage, neurologic dysfunction, infection were infrequent. Two patients presented with manifestations of endocrinopathies (Cushing syndrome, acromegaly). The tumor was discovered incidentally in 7 patients, during the evaluation of other pathology. Three patients were found to have Currarino syndrome (a triad of sacral agenesis, presacral mass, anorectal malformation). 
Imaging modalities including ultrasound, CT, and MRI were the primary modes of diagnosis in the majority of the cases. The median size of the tumor is $11.8 \mathrm{~cm}^{*} 9.7 \mathrm{~cm}$. Biochemical studies evaluating alpha-fetoprotein (AFP), human chorionic gonadotropins (HCG), carcinoembryonic antigen (CEA), lactate dehydrogenase (LDH) carcinoma antigen (CA 125), carbohydrate antigen 19-9 (CA-19-9) were rarely performed, and when performed were always negative. Preoperative biopsy was performed in 28 patients, with indeterminate findings in 16 patients, and concordant with pathology in 12 patients. Histopathologic analysis was conclusive in all the cases and is the gold standard. The surgical approach used was the posterior approach $(n=45)$, anterior approach $(n=12)$ and combined abdomino-perineal approach $(\mathrm{n}=10)$, and mostly single staged ( $\mathrm{n}$ =39). Resection of the coccyx and/ or sacrum was performed in 28 patients. Only 3 patients underwent a malignant transformation. Post-operative complications were very infrequent, and included pain $(n=4)$, infection $(n=3)$ and bladder dysfunction $(n=3)$. Interestingly, several long-standing cases reported were from developing countries and rural areas. The median follow-up time was 1.25 years.

Patients with Altman type IV category of benign SCT were the most frequent $(n=28)$, with a relatively short mean duration of symptoms ( 2 years) and presented mostly with pelvic pain $(\mathrm{n}=$ 11). The presacral tumor was smaller $(8 \mathrm{~cm} * 7.2 \mathrm{~cm})$ and was mostly resected by the posterior approach $(n=18)$. There were fewer patients with type II $(n=13)$ and type III $(n=9)$ benign SCTs. Type II cases presented mostly with mass $(n=9)$ and the patients mostly underwent resection by posterior approach $(n=7)$. Type I SCT was not identified even in a single adult. Other clinicopathologic characteristics were similar in other groups.

\section{Discussion}

Less than 75 total cases of benign SCT were reported over the last 70 years. Compared to pediatric cases of SCT, the clinicopathologic characteristics of these rare lesions remain poorly documented.

\section{Origins}

Teratomas are the most common type of germ cell tumors, and occur frequently in the ovaries, followed by testes, anterior mediastinum, retroperitoneum, and sacrococcygeal regions. SCTs are the commonest fetal neoplasms, while the adult SCTs might arise from the suggested to arise from multipotent cells in Henson's node, which migrated caudally to the sacrococcygeal region [2]. Other possible mechanisms include a mitotic proliferation of misplaced germ cells that have migrated from the yolk sac [4]. Adults cases of benign SCT often represent tumors that were present at birth (congenital), but not detected until adulthood. Several patients in our study might have had slow-growing tumors, which presented late with the manifestation of symptoms.

\section{Demographics}

SCTs occur with a prevalence of 1 in 30,000-70,000 with a female to male ratio of $4: 1$ [1]. The female preponderance was also seen in adult cases of benign SCT, and also within each Altman type. The female preponderance may be related to the delayed differentiation of the ovaries compared to testes [15]. We observed that the geographic distribution of cases is skewed towards developing regions of the world and in lower socioeconomic regions of the developed countries. The atypical longer presentation times are attributable to a delayed tendency in seeking medical care. However, these observations may be incorrect due to bias in reporting.

\section{Clinical presentation}

The clinical presentation of benign SCT depends upon the location and extension of the tumor and could be very diverse. The presenting symptoms in the fetus and newborn, are different from their adult counterparts. In the pediatric population, benign SCT may manifest as hydrops, polyhydramnios, fetal urinary obstruction, hemorrhage or rupture of the tumor, dystocia [29]. In adult cohorts, benign SCTs mostly present with low back pain or pelvic pain, mass, bowel, or bladder symptoms while neurological dysfunction, ulceration, and drainage were relatively rare. Incidental presentation is rare, and patients seek medical attention upon the manifestation of symptoms. Projection of the mass (Type II) externally may cause disfigurement and discomfort in addition to painful skin excoriation and ulcers. Repeated infections may result in perianal fistulas, abscess, cellulitis, or osteomyelitis [4]. Symptoms associated with cosmetic blemish and reduced quality of life [30] were poorly reported. Rarely, SCTs have been reported to have differentiated into limbs [6] or presented as endocrinopathies. In rare instances, benign SCT may occur as a component of Currarino 
syndrome. In patients with presacral SCT cases, the components of Currarino syndrome, including sacral agenesis and anorectal malformation should be carefully screened.

\section{Diagnosis}

Pediatric SCTs are diagnosed on prenatal ultrasound or at birth. Patients with no perinatal care or with very small SCTs that are missed in childhood may persist into adulthood. In adults, a diagnosis of SCT may be suspected during a routine pelvic or rectal examination that detects the presence of a mass or tumor. Patients with extrapelvic SCTs, who present late are likely asymptomatic or have limited access to medical care. Early diagnosis is important to prevent malignant transformation and complications. Imaging modalities such as transrectal or transvaginal ultrasound/computerized tomography, CT scan/magnetic resonance imaging, MRI are central to the diagnosis of these lesions and in delineating their origin, extent, and relation to the pelvic and abdominal organs. MRI is superior to CT in visualizing the various anatomic and pathologic components of SCT. Furthermore, imaging also guides operative management. The value of preoperative biopsy is not clear $[4,31]$. Serum tumor markers such as AFP, HCG, LDH, CA 125, CA 19-9 were infrequently performed, have less specificity, and have no value in the diagnosis or monitoring of benign SCTs. However, elevated markers may indicate malignant SCTs and can be valuable for monitoring the disease progression post-resection $[32,33]$.

Pathological specimens usually show derivatives from two or more germ layers and is indicative of the diagnosis. Histopathology is the gold standard diagnostic modality, to identify benign SCTs and differentiate them from their malignant counterparts. SCT may contain mature tissue that appears like any tissue in the body, or immature tissue resembling embryonic tissues. These tumors are usually cystic and benign with only a $1-2 \%$ chance for malignant transformation in adults. Cysts may be filled with serous fluid, mucoid, or sebaceous material and lined by true epithelium.

\section{Differential diagnosis}

A variety of additional tumors must be differentiated from individuals with SCT including lipomas, chordomas, soft tissue tumors, neurofibromas, hamartomas, neuroblastomas or tailgut cysts [2].

\section{Staging}

Staging is based on the Altman classification of SCTs depending on the anatomy of the tumor in relation to the body.

- $\quad$ Type I: Predominant extrapelvic SCT with minimal intrapelvic component

- $\quad$ Type II: Predominant extrapelvic SCT with significant intrapelvic component

- $\quad$ Type III: Predominant intrapelvic SCT with minimal extrapelvic component

- Type IV or presacral teratoma: Complete intrapelvic SCT with no extrapelvic component.

Intra-pelvic masses, Altman type IV tumors are common in adults, in contrast to neonates, where more than $90 \%$ present as extra pelvic masses [34]. The clinical presentation, duration of symptoms, and the size of the tumor were relatively different in type IV patients, compared to the other subtypes.

\section{Management}

The preferred treatment modality is complete surgical resection and is curative in the context of benign SCTs. Concomitant coccygectomy is usually performed to eliminate the risk of tumor recurrence [35]. Early surgical intervention normally results in a favorable long-term treatment outcome and good quality of life. Several adult patients in our cohort belonged to an underprivileged socioeconomic class which led to delay in seeking medical attention.

The surgical approach is based on tumor size, location, involvement of surrounding organs. Common surgical approaches include transabdominal, transsacral, or transperineal or a combination thereof. Recent advances allowed the use of laparoscopic-assisted approach for tumor resection [13]. The posterior transsacral approach with preservation of the rectum is used most often for SCT that extend below S3 while anterior approach or combined abdomino-perineal approach is used for tumors that extend above S3 [21]. An additional abdominal incision may be required if the SCT extends into the pelvis and the retroperitoneal space [8]. Complex cases may require multi-staged resection. 
Post-operative pain is the major surgical morbidity for the surgical excision of SCT. Massive bleeding may also complicate the resection; thus, meticulous dissection between the presacral fascia and the rectal fascia aids in the prevention of excessive blood loss and iatrogenic ureteral injuries. The vascular supply to SCTs should be cautiously controlled as they may share a common blood supply with vital pelvic organs [6]. Closure of large wounds may require flap reconstruction.

\section{Prognosis}

The prognosis for benign SCT is excellent, and malignant transformation is very rare. The age at diagnosis, histology, coccygectomy, and the extent of intrapelvic extension are important prognostic factors [6].

\section{Conclusion}

Benign SCTs remain rare lesions with unique clinicopathologic characteristics. Differentiation of benign entities from the malignant counterparts, and early intervention is central to the management of these lesions. Complete resection without tumor disruption that includes the coccyx, but sparing other surrounding structures, should result in a low recurrence rate. Multidisciplinary care is advised in adults patients with sacrococcygeal teratomas to optimize outcomes.

\section{Authors' Contributions}

AY(Anudeep Yekula), KKY conceptualized the review. AY(Anudeep Yekula), SI, AK, AP, AY(Anuroop Yekula) performed the literature review and data analysis. All authors participated in study design, critical analysis of systematic literature review, and manuscript writing.

\section{Conflict of Interest}

None.

\section{Funding}

None.

\section{Bibliography}

1. Kremer M E B., et al. "Quality of life in adulthood after resection of a sacrococcygeal teratoma in childhood: a Dutch multicentre study". Archives of Disease in Childhood - Fetal and Neonatal Edition 100 (2015): F229-232.
2. Luk S Y., et al. "Sacrococcygeal teratoma in adults: case report and literature review". Hong Kong Medical Journal 17 (2011): 417-420.

3. Zhang F., et al. "Mucinous tumor arising in a giant sacrococcygeal teratoma: A rare case report. Medicine 96 (2017): e8759.

4. Sheng QS., et al. "Multi-stage resection and repair for the treatment of adult giant sacrococcygeal teratoma: A case report and literature review". Oncology Letter 10 (2015): 425-429.

5. Diaz-Aguilar D., et al. "Adult sacrococcygeal teratoma with coccygectomy: A case report with a review of the literature". Surgical Neurology International 8 (2017): 260.

6. Yekula MSKK and Yekula A. "Adult Sacrococcygeal Teratoma: The third leg: A rare case report in a 25 year old man". International Journal of Surgery Case Reports 14 (2015): 146-148.

7. Babiker T., et al. "Acromegaly and Cushing's syndrome caused by a neuroendocrine tumor arising within a sacrococcygeal teratoma". Clinical Case Report 5 (2017): 1768-1771.

8. Xu XM., et al. "Adult sacrococcygeal teratoma: a retrospective study over eight years at a single institution". Journal of Zhejiang University Science B 20 (2019): 670-678.

9. Kumar N., et al. "Sacro-coccygeal teratoma in adult: Two rare case reports and review of literature". International Journal of Applied and Basic Medical Research 4 (2014): 122-124.

10. Shatnawi $\mathrm{N} \mathrm{J.,} \mathrm{et} \mathrm{al.} \mathrm{"A} \mathrm{giant} \mathrm{sacrococcygeal} \mathrm{teratoma} \mathrm{in} \mathrm{adult}$ female: A case report". International Journal of Surgery Case Reports 54 (2019): 47-50.

11. Sukhadiya M V and Das U. "Laparoscopic Approach to Type IV Sacrococcygeal Teratoma in an Adult". Indian Journal of Surgery 77 (2015): 62-63.

12. Afuwape 0 0., et al. "Adult sacrococcygeal teratoma: a case report". Ghana Medical Journal 43 (2009): 40-42.

13. Szyllo K and Lesnik N. "Sacrococcygeal teratoma - case report and review of the literature". American Journal of Case Reports 14 (2013): 1-5. 
14. Mourad A P., et al. "A case of an asymptomatic sacrococcygeal teratoma diagnosed in adulthood". Surgical Case Reports 2020 (2020): rjaa462.

15. Miles R M., et al. "Sacrococcygeal teratomas in adult". Annals of Surgery 179 (1974): 676-683.

16. Ng E W., et al. "Sacrococcygeal teratoma in adults: case reports and a review of the literature". Cancer 86 (1999): 1198-1202.

17. Conklin J and Abell M R. “Germ cell neoplasms of sacrococcygeal region”. Cancer 20 (1967): 2105-2117.

18. Tolins S H and Cooper P. "Presacral teratoma”. American Journal of Surgery 115 (1968): 734-737.

19. Wilkinson J M., et al. "Obstructed defaecation because of adult sacrococcygeal teratoma”. Lancet 340 (1992): 1287-1288.

20. Emoto, S., et al. "Surgical management for a huge presacral teratoma and a meningocele in an adult with Currarino triad: a case report". Surgical Case Reports 4 (2018): 9.

21. Simpson P J., et al. "Surgical outcomes in adults with benign and malignant sacrococcygeal teratoma: a single-institution experience of 26 cases". Diseases of the Colon and Rectum 57 (2014): 851-857.

22. Al-Essa AA., et al. "Adult sacrococcygeal teratomas". Saudi Medical Journal 25 (2004): 367-369.

23. Audet I M., et al. "Adult sacrococcygeal teratomas". American Surgery 66 (2000): 61-65.

24. Moreno-Fernández, J., et al. "Corticotrophin-dependent Cushing syndrome due to Sacrococcygeal Teratoma detected by [18F]fluorodeoxyglucose positron emission tomography". The Journal of Clinical Endocrinology and Metabolism 93 (2008): 3282-3283.

25. Killen D A and Jackson LM. "Sacrococcygeal teratoma in the adult". Archives of Surgery 88 (1964): 425-433.

26. Pye G and Blundell J W. "Sacrococcygeal teratoma masquerading as fistula-in-ano". Journal of the Royal Society of Medicine 80 (1987): 251-252.
27. Jucá M., et al. "Sacrococcycygeal teratoma in adult: report of a case". International Journal of Gastrointestinal Cancer 37 (2006): 91-93.

28. Tsutsui A., et al. "Successful laparoscopic resection of a sacrococcygeal teratoma in an adult: report of a case". Surgery Today 41 (2011): 572-575.

29. Woodward P J., et al. "From the archives of the AFIP: a comprehensive review of fetal tumors with pathologic correlation". Radiographics 25 (2005): 215-242.

30. Issues in Pediatric and Adolescent Medicine Research and Practice: 2011 Edition. Scholarly Editions, (2012).

31. Pendlimari R., et al. "Rare malignant neuroendocrine transformation of a presacral teratoma in patient with Currarino syndrome". International Journal of Colorectal Disease 25 (2010): 1383-1384.

32. Tjalma W A A. "The value of AFP in congenital cervical teratoma". Journal of Pediatric Surgery 38 (2003): 1846.

33. Chen C., et al. "[Diagnostic value of multiple tumor marker detection for mature and immature teratoma of the ovary]". $A i$ Zheng 27 (2008): 92-95.

34. Mindaye E T., et al. "Adult case of giant sacrococcygeal teratoma: case report”. BMC Surgery 20 (2020): 295.

35. Wishnia SC., et al. "Management of a presacral teratoma in an adult". Journal of Clinical Oncology 26 (2008): 2586-2589.

\section{Volume 4 Issue 6 June 2021} (C) All rights are reserved by Anudeep Yekula., et al. 